\title{
System-level performance of C-NOMA: a cooperative scheme for capacity enhancements in 5G mobile networks
}

\section{Marcano, Andrea; Christiansen, Henrik Lehrmann}

Published in:

2017 IEEE 86th Vehicular Technology Conference (VTC-Fall)

Link to article, DOI:

10.1109/VTCFall.2017.8288415

Publication date:

2017

Document Version

Peer reviewed version

Link back to DTU Orbit

Citation (APA):

Marcano, A., \& Christiansen, H. L. (2017). System-level performance of C-NOMA: a cooperative scheme for capacity enhancements in 5G mobile networks. In 2017 IEEE 86th Vehicular Technology Conference (VTC-Fall) IEEE. https://doi.org/10.1109/VTCFall.2017.8288415

\section{General rights}

Copyright and moral rights for the publications made accessible in the public portal are retained by the authors and/or other copyright owners and it is a condition of accessing publications that users recognise and abide by the legal requirements associated with these rights.

- Users may download and print one copy of any publication from the public portal for the purpose of private study or research.

- You may not further distribute the material or use it for any profit-making activity or commercial gain

- You may freely distribute the URL identifying the publication in the public portal 


\title{
System-level performance of C-NOMA: a cooperative scheme for capacity enhancements in 5G mobile networks
}

\author{
Marcano, Andrea; Christiansen, Henrik Lehrmann \\ Published in: \\ 2017 IEEE 86th Vehicular Technology Conference (VTC-Fall) \\ Link to article, DOI: \\ 10.1109/VTCFall.2017.8288415 \\ Publication date: \\ 2017 \\ Document Version \\ Publisher's PDF, also known as Version of record \\ Link back to DTU Orbit
}

\section{Citation (APA):}

Marcano, A., \& Christiansen, H. L. (2017). System-level performance of C-NOMA: a cooperative scheme for capacity enhancements in 5G mobile networks. In 2017 IEEE 86th Vehicular Technology Conference (VTC-Fall) IEEE. DOI: 10.1109/VTCFall.2017.8288415

\section{DTU Library}

\section{Technical Information Center of Denmark}

General rights

Copyright and moral rights for the publications made accessible in the public portal are retained by the authors and/or other copyright owners and it is a condition of accessing publications that users recognise and abide by the legal requirements associated with these rights.

- Users may download and print one copy of any publication from the public portal for the purpose of private study or research.

- You may not further distribute the material or use it for any profit-making activity or commercial gain

- You may freely distribute the URL identifying the publication in the public portal 


\title{
Sytem-level performance of C-NOMA: a cooperative scheme for capacity enhancements in $5 \mathrm{G}$ mobile networks
}

\author{
Andrea S. Marcano and Henrik L. Christiansen \\ DTU Fotonik \\ Technical University of Denmark \\ 2800 Kongens Lyngby, Denmark \\ anmarc@ fotonik.dtu.dk; hlch@ fotonik.dtu.dk
}

\begin{abstract}
Non-orthogonal Multiple Access (NOMA) is a promising scheme for improving the capacity of $5 \mathrm{G}$ networks. Since not all the users (UEs) in a cell are good candidates for NOMA, hybrid multiple access (MA) systems where NOMA and the typical orthogonal multiple access (OMA) schemes are combined, are expected. In this paper we propose cooperative NOMA (C-NOMA), which is based on cooperative scheduling and load balancing among a group of cells. Results from our system-level performance analysis show that in scenarios where only some cells are fully loaded, C-NOMA offers up to 3.5-fold increase in the UEs throughput compared to non-cooperative NOMA (NC-NOMA). Moreover, the cells experienced a capacity increase between $12-18 \%$. Therefore, C-NOMA is an appealing scheme for improving the capacity in groups of cells with very unequal load, e.g. hotspot areas.
\end{abstract}

Keywords- cooperative NOMA; NOMA; hybrid MA; 5G; capacity; throughoput

\section{INTRODUCTION}

The continuing growth in the demand of both the users (UEs) data rates and the network capacity has been the main driver for the mobile networks evolution. This still remains as one of the key motivations for the deployment of fifth generation $(5 \mathrm{G})$ mobile networks. Higher capacities can be achieved through cell densification, more spectrum, and a more effective use of the available resources. To target the latter, Non-orthogonal multiple access (NOMA) has been considered as a multiple access (MA) scheme to be implemented in $5 \mathrm{G}$ networks [1]-[4].

NOMA offers advantages over the typical orthogonal multiple access (OMA) schemes that have been used so far in mobile networks. Unlike orthogonal frequency multiple access (OFDMA) used in 4G networks, NOMA additionally multiplexes UEs in the power domain based on their channel conditions, giving access to the UEs with strong channel conditions to the resources allocated to the UEs with poor channel conditions. This allows for a higher spectral efficiency in scenarios where the available resources become scarce. Nevertheless, not all the UEs being served by a cell are candidates to be multiplexed in the power domain and have their signal sent using NOMA [5]. Therefore, hybrid MA systems where OMA and NOMA are combined are expected [2], [6]. Most of the research work done on NOMA and its impact in the system capacity has been focused in the performance of NOMA as the only MA scheme [7]-[9]. The performance evaluation of hybrid MA systems is therefore highly anticipated.

The work in [6] focused on the performance of such hybrid MA system in a single cell, showing a significant gain in the overall system capacity and the UEs data rates. In this article, we focus on the implementation of a hybrid MA system in a typical scenario considered for $5 \mathrm{G}$ networks - a cluster of small cells deployed in a hotspot area [10]. For this, we propose the implementation of a cooperative NOMA (C-NOMA) scheme, based on load balancing and cooperative scheduling among a group of cells with unequal load. The main idea of C-NOMA is to increase the system capacity by maximizing the number of NOMA UEs in highly loaded cells and thus reusing the resources more efficiently, in comparison to a hybrid MA system where non-cooperative NOMA (NCNOMA) is implemented.

\section{NOMA BASICS}

The principle behind NOMA consists on selecting UEs with large channel gain difference to be multiplexed in the same time/frequency resources; this difference in the channel gain then translates into multiplexing gain [3]. Superposition transmission schemes and adaptive power allocation in the transmitter are implemented to multiplex the signals. The power ratio assigned to an allocated UE will depend on its channel conditions; the lower the channel gain, the higher the power ratio. Due to the extra interference that is intentionally added in the transmitter with NOMA, interference cancellation (IC) techniques are used in the receiver side.

The achievable data rates for each UE paired with NOMA can be calculated as follows:

$$
\mathrm{R}_{\mathrm{NOMA}}=\mathrm{B} * \beta * \log _{2}(1+(\alpha * \operatorname{SINR}))
$$

where $\beta$ is the portion of the total bandwidth $B$ ocuupied by the UE and $\alpha$ represents the portion of the power allocated. In comparison, the data rates for OMA are calculated as:

$$
\mathrm{R}_{\mathrm{OMA}}=\mathrm{B} * \beta * \log _{2}(1+\mathrm{SINR})
$$

Although the term $\alpha$ in equation 1 reduces the argument of the logarithm in the NOMA case, this term does not affect the result as much as the $\beta$ factor outside the logarithm [2]. This is the reason for the higher data rate in NOMA, since $\beta$ would be larger in NOMA because of the bandwidth increase thanks to the pairing. 


\section{HYRBID MULTIPLE ACCESS SYSTEMS}

The use of NOMA in radio access is not a simple implementation. In NOMA, extra interference is intentionally added to the signal; hence, the SINR needed to correctly decode a NOMA signal is higher than that for OMA [5]. Mechanisms, such as modulation and coding scheme (MCS) adjustments and extra transmission power, can be implemented to compensate for the decrease in the SINR [6], [11] and keep the block error rate (BLER) below the set value. Therefore, the scenarios in which NOMA results an interesting implementation should be defined.

A fully loaded cell, for example, is a scenario in which NOMA helps increase the system performance, whether this is measured as the number of UEs served within certain time and with certain resources, or as the increase in the UEs and/or system throughput. In a scenario where there is no congestion in the cell and therefore each UE can have access to the needed resources, the implementation of NOMA would only make the signal transmission and reception more complex. Let us assume that we have two UEs with a high difference in their channel gain, and each one only requires half of the available resources for the on-going transmission; both UEs qualify as NOMA candidates to be paired, but such pairing would not be necessary since each UE already has access to the required resources.

Nevertheless, scenarios where NOMA is required would be highly probable in 5G networks. When implementing NOMA it must be considered that not all the UEs should be paired; besides the channel gain difference, the modulation orders of the UEs to be paired also has to be considered. The work in [5] explains that, for two paired UEs, if the modulation order of the UE with the lower channel gain is higher than two (corresponding e.g. to QPSK modulation), the superposed constellation becomes too complex to decode. These constraints in the characteristics of a possible pair with NOMA lead to a hybrid MA system, where both OMA and NOMA can be combined in every subframe, depending on the cell load conditions and the UEs channel conditions.

The work in [6] presents a performance analysis of a fully loaded cell with a hybrid MA system, where the MCS and transmission power are adjusted for the NOMA UEs. Results show that in such a system configuration up to 1.78-fold increase in the system capacity can be achieved when adding NOMA as an option. The UEs throughput also increased up to 3.31-fold and an extra transmission power between $14 \%$ and $19 \%$ was needed to keep the BLER below $10 \%$.

\section{PRoposed COOPERATIVE NOMA (C-NOMA) SCHEME FOR HYRBID MULTIPLE ACCESS SYSTEMS}

In a hybrid MA system, the capacity could even be further increased if more UEs could be paired in the fully loaded cells. When resources are scarce, the higher the number of NOMA UEs, the higher the system capacity because of the resource reuse. Therefore, the scheduling process in a fully loaded cell should be optimized aiming at pairing as many UEs as possible, and keeping the OMA UEs to a minimum.

As an approach for such optimization, we propose C-NOMA, a cooperative scheduling and load balancing scheme among a group of cells with unequal load; the main idea behind this cooperation is to take advantage of the inter cell interference found on the edges of the cells. If a UE A that is located between the overlapping edges of two cells cannot be paired in its fully loaded serving cell (home cell), perhaps a neighbor interfering cell (target cell) has either enough free resources or/and a good pair for UE A; if so a forced handover is performed for UE A from the home cell to the target cell.

To illustrate the C-NOMA scheme, let us assume that we have two cells, Cell 1 with three UEs and Cell 2 with two UEs, as shown in Fig. 1 (a), and that the scheduling for subframe $\mathrm{N}$ is currently ongoing. Cell 1 is fully loaded while Cell 2 has available resources. NOMA, with a maximum of two multiplexed UEs per pair, is then implemented in Cell 1 and UE A and UE B are paired, leaving UE C with no pair; in Cell 2, there is no need to use NOMA. As UE C is located at the edges of both cells, Cell 1 exchanges UE $\mathrm{C}$ information with Cell 2, aiming at reducing the OMA UEs and have more resources for the NOMA UEs (i.e. UE A and UE B), as shown in Fig. 1 (b). Cell 2 then determines if it can allocate UE C; at this point, two options arise, Fig. 1 (c):

- Option 1 - UE C with OMA in Cell 2: there are enough free resources for UE C in Cell 2 so there is no need to implement NOMA, or UE C cannot be paired in Cell 2. In either case, the resources can be allocated to UE C.

- Option 2 - UE C with NOMA in Cell 2: UE C needs more resources than those available in Cell 2 and there is a suitable pair for it.

Once the cooperative scheduling is done, the definitive resource allocation is performed. Cell 2 indicates to Cell 1 that it can schedule UE $\mathrm{C}$ and then a forced handover is performed - Cell 1 sends handover signaling to UE C without such action being triggered by handover events reported by UE C. Since the forced handover needs to be indicated to UE $\mathrm{C}$ in the current subframe $\mathrm{N}$, UE $\mathrm{C}$ would not be allocated any data resources until subframe $M$, with $M>N$, in its new serving cell, Cell 2.

The implementation of C-NOMA is most beneficial when the target cell for the cooperative scheduling and forced handover is not fully loaded and has, therefore, some available resources. Otherwise the throughput of the UEs in the target cell would be affected, which at the ends could generate insignificant or no capacity gain to the system.

C-NOMA is an attractive implementation to enhance the system capacity in e.g. for a hotspot area, where some cells are highly loaded while the neighbor cells are not. The load balancing principle has been studied before for the current networks [12] and is also implemented nowadays in hotspot areas. However, with C-NOMA not only can the load be 


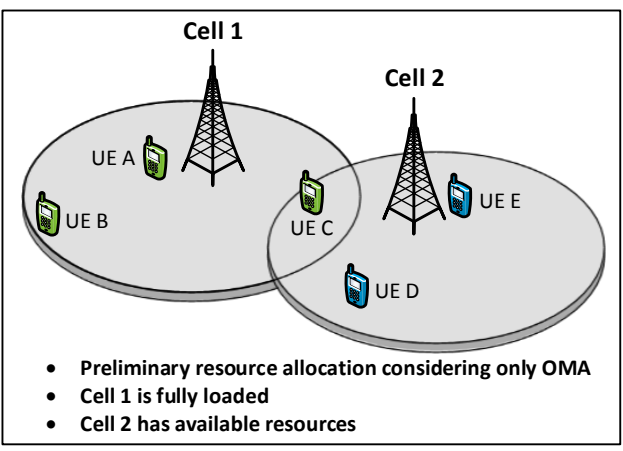

(a)

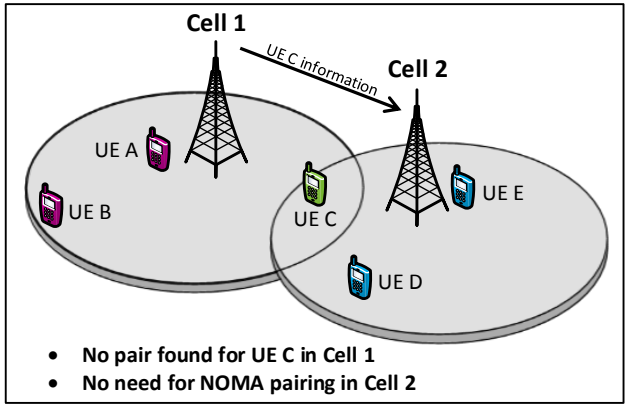

(b)
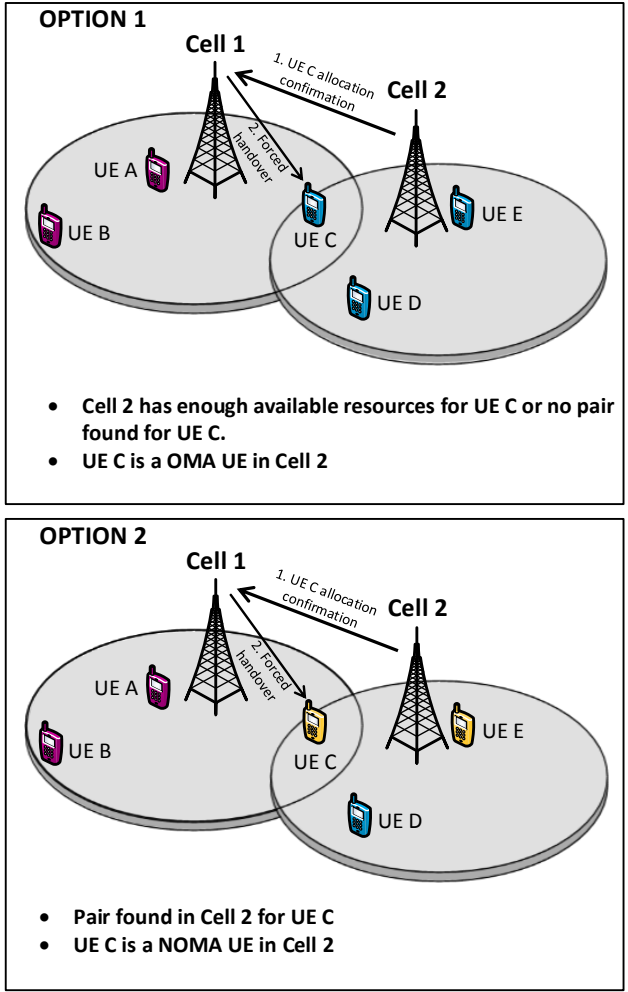

(c)

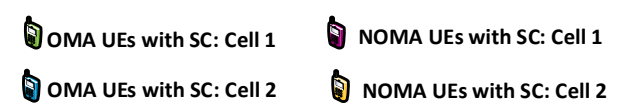

Fig. 1 C-NOMA scheme proposed aiming at reducing the OMA UEs in fully loaded cells. (a) Preliminary resource allocation only with OMA; (b) Exchange information of OMA UEs that cannot be paired in fully loaded cells; (c) Possible options of C-NOMA: the incoming UE can either be an OMA or NOMA UE in target cell. balanced in the system, but also the system capacity can be increased; this can be achieved by minimizing the OMA UEs and increasing the resources for NOMA UEs in a cell where resources are scarce, and therefore a more effective reuse of the resource is highly needed. An improvement in the overall system performance is expected from C-NOMA in comparison to NC-NOMA for a group of cell with unequal load, since free resources can be utilized more efficiently.

\section{PERFORMANCE ANALYSIS OF C-NOMA}

The work presented in this paper focuses on the capacity and throughput gain of C-NOMA over NC-NOMA. The system was modeled in MATLAB using the LTE Toolbox. A wrap-around model of seven clusters with seven cell sites each (with three cells per site) was used to model the network.

The context assumed for the simulations is a hotspot area being covered by small cells, aiming at increasing the system capacity in such area. The carrier frequency was selected in the mmWave band because of the large amount of available spectrum and its promising use in $5 \mathrm{G}$ networks for the small cells. The following model was used to estimate the path losses [13]:

$$
\begin{gathered}
P_{L O S}=69.8 d B+20 \log (d)+x_{\sigma} \\
P_{N L O S}=69.8 d B+33 \log (d)+x_{\sigma}
\end{gathered}
$$

where $x_{\sigma}$ represents the shadowing factor and it is a random Gaussian variable with mean zero and standard deviation $\sigma=5.2 \mathrm{~dB}$ for line of sight (LOS) and $\sigma=7.6 \mathrm{~dB}$ non line of sight (NLOS). The probability of LOS was set as 0.5 . No small scale model was considered during the simulations, since there is not yet a defined Power Delay Profile (PDP) for mmWave. Table 1 summarizes the propagation parameters.

\begin{tabular}{|c|c|c|}
\hline & OMA & NOMA \\
\hline Carrier Frequency & \multicolumn{2}{|r|}{$73 \mathrm{GHz}$} \\
\hline Channel Bandwidth & \multicolumn{2}{|c|}{$100 \mathrm{MHz}$} \\
\hline Modulation Scheme & \multicolumn{2}{|c|}{ QPSK, 16QAM, 64QAM, 256QAM } \\
\hline Maximum Tx Power & \multicolumn{2}{|c|}{$10 \mathrm{~mW}$} \\
\hline \multirow{2}{*}{$\begin{array}{c}\text { Tx power allocation } \\
\text { per UE }\end{array}$} & \multirow{2}{*}{1} & $\begin{array}{c}0.25 \text { for UE with } \\
\text { higher channel gain }\end{array}$ \\
\hline & & $\begin{array}{c}0.75 \text { for UE with } \\
\text { lower channel gain }\end{array}$ \\
\hline Transmission mode & \multicolumn{2}{|r|}{ SISO } \\
\hline Tx gain & \multicolumn{2}{|r|}{$25 \mathrm{dBi}$} \\
\hline Rx gain & \multicolumn{2}{|r|}{$0 \mathrm{dBi}$} \\
\hline Noise factor & \multicolumn{2}{|r|}{$6 \mathrm{~dB}$} \\
\hline
\end{tabular}

\section{Table 1 Propagation parameters for the system modeled}

Two scenarios were considered for the simulations; in the first one, the cell sites are unequally loaded; for simplicity reasons, all the cells belonging to the same site are assumed to have the same load. In the second scenario, all cell sites are fully loaded, therefore the target cell does not have available resources for more UEs; however it can still try to find pairs for the incoming UEs. The load for the cells was determined based on the number of available physical resource blocks (PRBs), with zero free PRBs corresponding to a load of $100 \%$. Table 2 summarizes the characteristics of each scenario for one 
cluster, with cells sites 1 and 6 fully loaded, 5 and 7 medium loaded, and 2-4 lightly loaded in Scenario 1. Fig. 2 shows the grid configuration of one cluster and its direct neighbors for each scenario.

Table 2 Load for each cell site in a cluster for the two scenarios defined

\begin{tabular}{|c|c|c|}
\cline { 2 - 2 } \multicolumn{1}{c|}{} & \multicolumn{2}{c|}{ Average load (\%) } \\
\hline Cell Site & Scenario 1 & Scenario 2 \\
\hline $\mathbf{1}$ & 100 & \multirow{1}{*}{} \\
\hline $\mathbf{2}$ & \multirow{2}{*}{30} & \multirow{2}{*}{100} \\
\hline $\mathbf{3}$ & 30 \\
\hline $\mathbf{4}$ & & \\
\hline $\mathbf{5}$ & 60 & \multirow{2}{*}{} \\
\hline $\mathbf{6}$ & 100 & \\
\hline $\mathbf{7}$ & 60 & \\
\hline
\end{tabular}

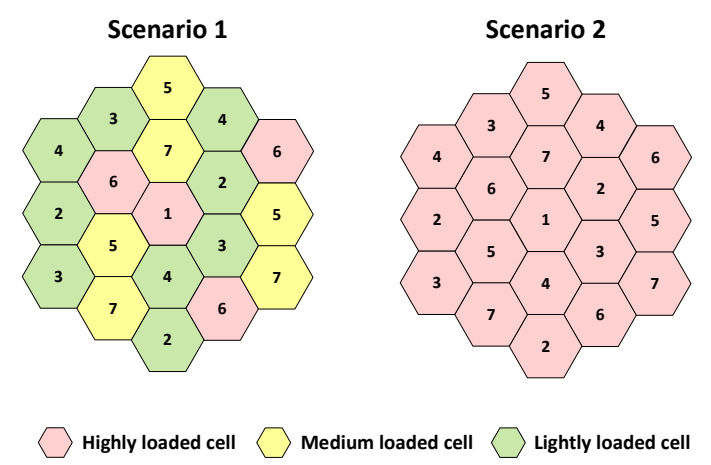

Fig. 2 Grid configuration of one cluster and its direct neighbors for the scenarios defined for the evaluation of C-NOMA

The UEs were placed randomly within the coverage area of the cell sites following a uniform distribution, with a maximum of 20,12, and 6 UEs for the fully, medium and lightly loaded cell sites, respectively. The packet size for the UEs was selected randomly, while guarantying that the load of the serving cell would not exceed the set value. No mobility was considered for simplicity reasons.

In the scheduler, for each cell a preliminary resource allocation is first done considering only OMA; in the fully loaded cells, the UEs transmitting in each subframe are grouped according to their modulation order and a proportional allocation was performed depending on the number of UEs in each group; a minimum of two allocated PRB was established. Then, if necessary, NOMA is implemented with a maximum of two multiplexed UEs per pair. From now onwards, we refer to each group of two multiplexed UEs in NOMA as a NOMA pair.

The process for finding the NOMA pairs is based on an exhaustive search. For this search UEs are grouped according to the modulation, as shown in Fig. 3. For our model we consider the modulations QPSK, 16QAM, 64QAM, and 256QAM, which leads to four groups. Each group is assigned a value of $n=2^{m}$ depending on the modulation order, with $m$ equal to $2,4,6$, and 8 , respectively. The search is done between two groups at a time, and all group combinations are considered. In this way it is guaranteed that the values of $m$ in a NOMA pair will not be the same. For our model a total of six group combinations were possible, and therefore six search iterations were defined (Fig. 3). The first search is done between the two groups with higher difference in the $n$ values, $\Delta n$ (e.g. Group 1 and Group 4, with $\Delta n=252$ ). During the search between two groups, each UE in the group with higher $m$ is evaluated with each unpaired UE in the other group. A NOMA pair is found when the following conditions are met:

- Condition 1: the throughput of both UEs must not be degraded. The methodology proposed in [6] was used for this purpose, based on MCS adjustments that need to be considered when switching from OMA to NOMA.

- Condition 2: one of the UEs must have $m=2$ (e.g. QPSK modulation), after performing the MCS adjustment.

After evaluating all possible pairs between two groups, the process continues with the other group combinations in a descending order according to $\Delta n$ (Fig. 3). When all the group combinations are evaluated, the search for NOMA pairs in the cell ends; this pairing search process gives priority to the NOMA pairs with higher channel difference.

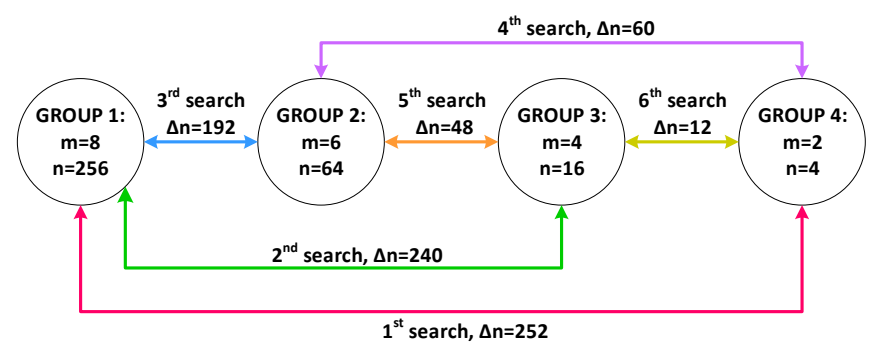

Fig. 3 Pairing search process used in the model

Once the NOMA pairs have been found, the implementation of C-NOMA starts for the OMA UEs located in the overlapping areas of neighboring cells. If one UE is located in the overlapping area of more than one cell, all the interfering cells participate in the allocation of such UE. The best target cell will be that one that offers the highest throughput for the UE in question, as shown in Fig. 4.

\section{RESULTS}

The performance of the system was evaluated in terms of the capacity and UEs throughput improvements, using an only OMA system as a benchmark. The results are shown only for one cluster of seven cell sites. First, Scenario 1 was evaluated; Fig. 5 shows the capacity gain for the cell sites, where we can see how C-NOMA significantly increases the capacity for all the cell sites. With C-NOMA the maximum gain was experienced by the fully loaded cells with an average of $88 \%$. This significant gain is due to the use of NOMA when resources are scarce while minimizing the OMA UEs. The less loaded cells (cells 2-4) experienced an average gain of $16 \%$ while the medium loaded cells (cells 5 and 7) experienced an average gain of $12 \%$. For NC-NOMA the fully loaded cells experienced an average gain of $75 \%$, while the cells that were not fully loaded did not experienced any gain; this was expected since if there are enough resources for all UEs there is no need to apply NOMA. 

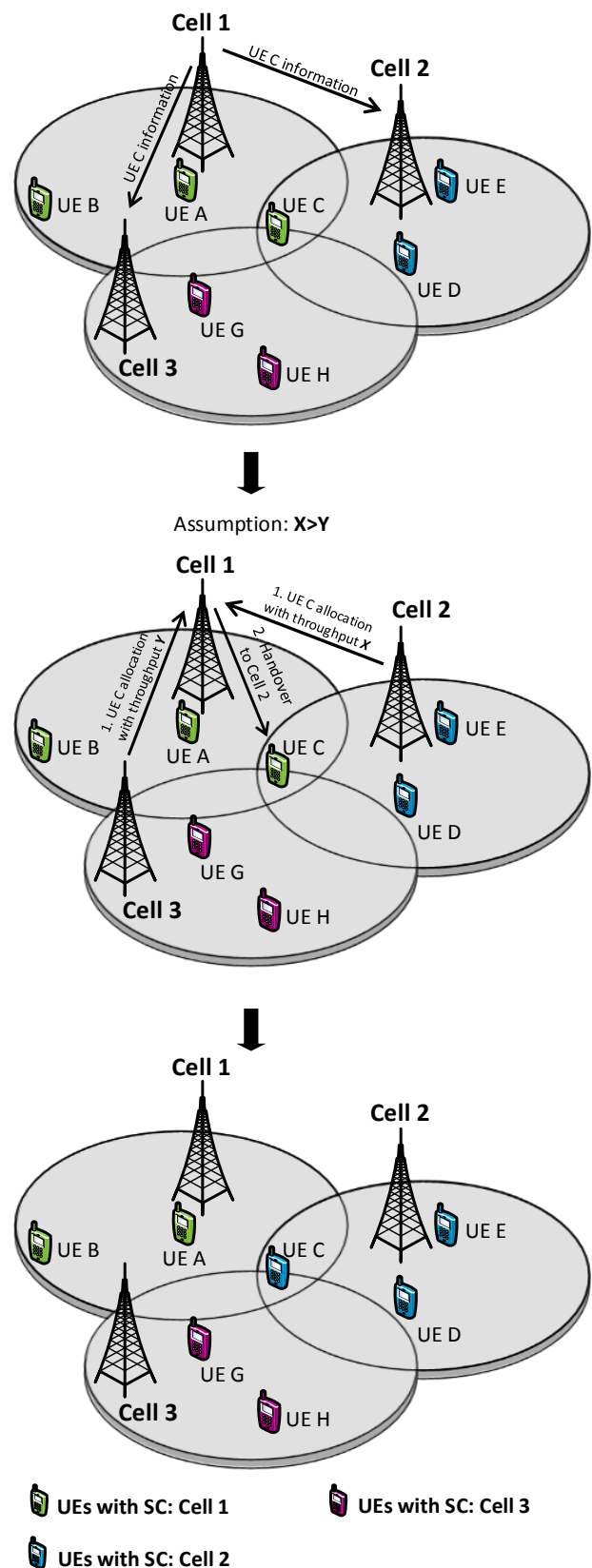

Fig. 4 C-NOMA functioning when the OMA UE in evaluation is receiving interference from more than one neighbor cell.

These results verify that when C-NOMA is implemented in a group of cells with unequal load, a capacity gain can always be expected. With C-NOMA the system resources are used more effectively by minimizing the OMA UEs and increasing the resources for NOMA pairs in loaded cells, while having the less loaded cells help in balancing the load. In overall, C-NOMA offered between $12-18 \%$ capacity gains for the cells in comparison to NC-NOMA.

To analyze the benefits of C-NOMA in the UEs throughput, Fig. 6 shows the PDF and CDF for the throughput gain. Here we can see that an UE has approximately $42 \%$ probability of increasing its data rate when C-NOMA is implemented instead of NC-NOMA, approximately $46 \%$ of maintaining its data rate and only approximately $12 \%$ of decreasing it. Moreover, we can see from the CDF that $90 \%$ of the UEs experienced a gain of 1.6-fold or below, with the remaining $10 \%$ experiencing high gain above 2 -fold increase. With these results it can be confirmed that C-NOMA is also an attractive implementation from most of the UEs perspective in the network.

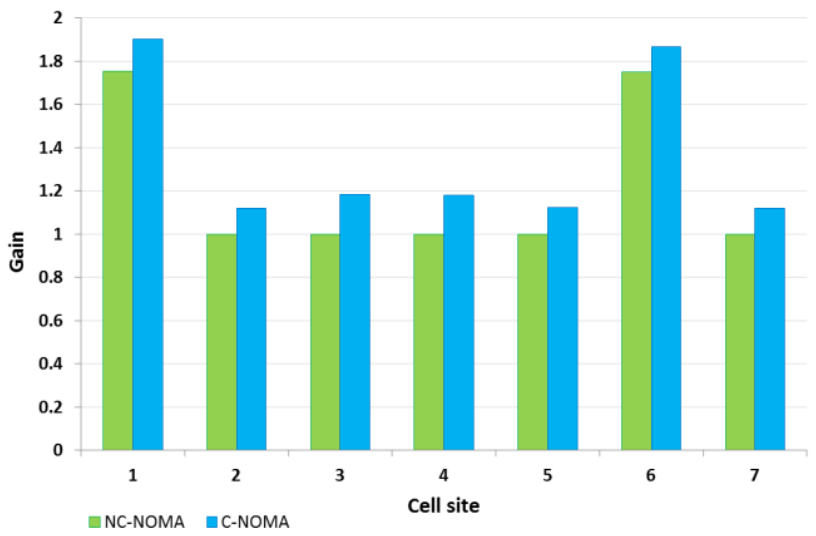

Fig. 5 Channel capacity gain for cell sites in Scenario 1

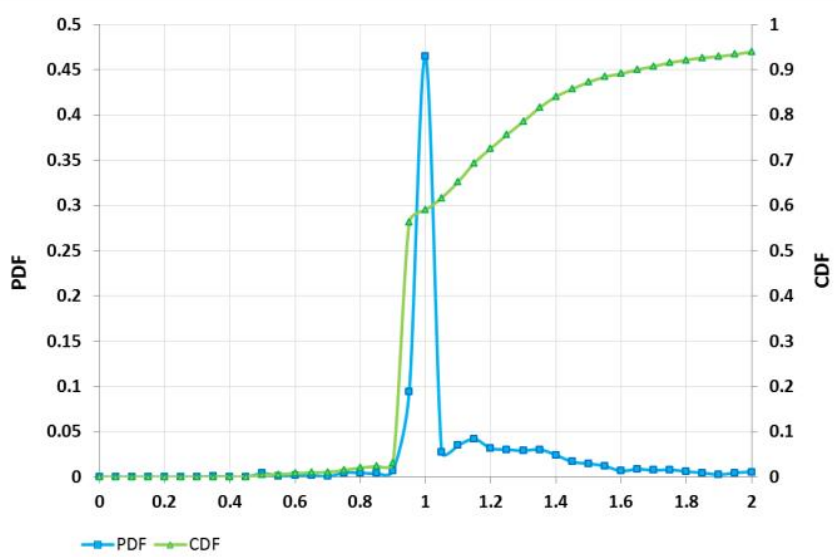

Fig. 6 UEs throughput gain of C-NOMA over NC-NOMA for Scenario 1

If we now analyze the performance of C-NOMA in Scenario 2, we can refer to Fig. 7, where it can be seen that the cells either had no capacity improvement in comparison to NC-NOMA or even experienced a capacity loss with an average of 2.5-fold decrease. Although C-NOMA still offers a considerably higher system capacity in comparison to OMA, with up to 1.7-fold increase, NC-NOMA offers higher capacity and less complexity in groups of cells with fully loaded cells.

Fig. 8 shows the PDF and the CDF for the UEs throughput gain in the cluster for Scenario 2. It can be seen that the highest probability corresponds to $27 \%$ for a gain of 0.95 , which translates to a decrease in the UEs throughput. A UE in this scenario has $20 \%$ probability of increasing its data rate and $23 \%$ probability of maintaining it, while it has $57 \%$ probability of decreasing it. From the CDF we can see that the highest increase in the throughput was of approximately 1.35- 
fold and that $80 \%$ of the UEs experienced no gain or a decrease in the throughput. These results show that C-NOMA in a scenario with all the cells in under the same load conditions has a higher probability of damaging the UEs throughput rather than increasing it. The reason for this behavior is that since the cells have no available resources, taking UEs from other cells, even if it is to pair them, requires resources that need to be taken away from other UEs. Eventually, what is a gain in one cell can be a loss in another. Therefore for scenarios were all the cells have equal load, the implementation of C-NOMA would not offer a good tradeoff between system capacity and implementation complexity. Thus, under these conditions, it is preferable to implement NC-NOMA.

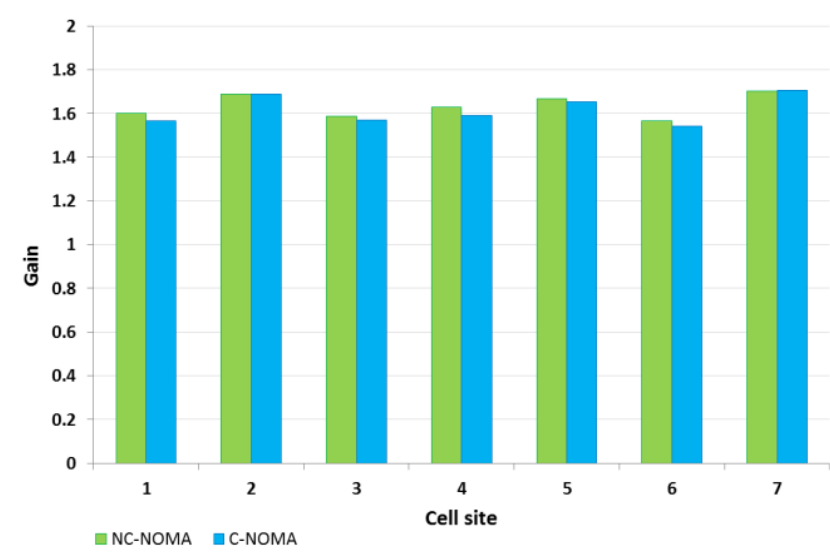

Fig. 7 Channel capacity gain for cell sites in Scenario 2

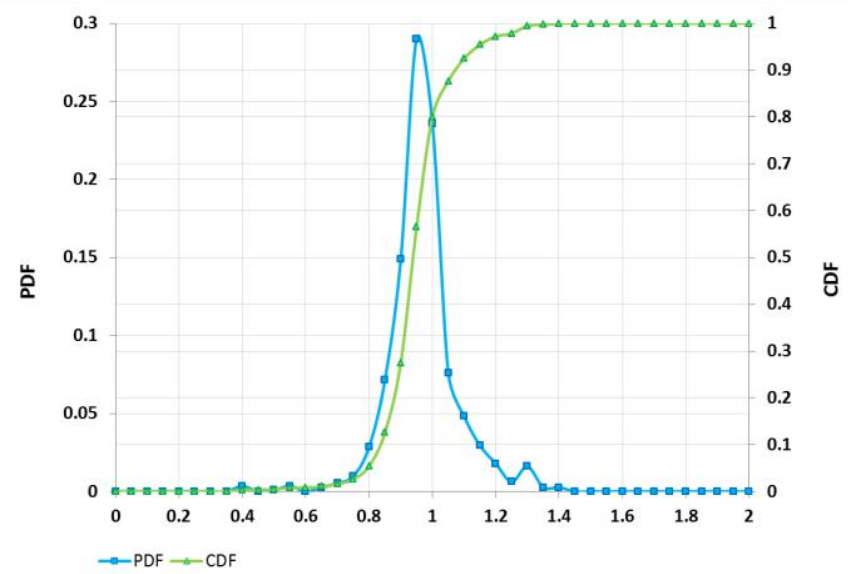

Fig. 8 UEs throughput gain of C-NOMA over NC-NOMA for Scenario 2

\section{CONCLUSIONS}

In this paper we proposed cooperative NOMA (C-NOMA), which is based on cooperative scheduling among neighboring cells and load balancing, aiming at enhancing the system capacity by minimizing the OMA UEs and increasing the resources for NOMA pairs, in groups of cells with unequal load. To evaluate the performance of C-NOMA, a cluster of seven cell sites (with three cells per site) was modeled; a comparison with the performance of non-cooperative NOMA
(NC-NOMA) was done. Results showed that when the cell sites are unequally loaded higher capacity was obtained for all cells, with a gain between $12-18 \%$ of C-NOMA over NCNOMA. From the UEs perspective, a $42 \%$ probability of increasing their throughput when implementing C-NOMA instead of NC-NOMA was obtained; moreover, they had $46 \%$ probability of increasing their throughput, and only $12 \%$ probability of decreasing it. These results confirmed that $\mathrm{C}$ NOMA has the potential to enhance the capacity in a system, for both the cells and the UEs, when the cells have different load conditions, e.g. in hotspot areas, which are highly anticipated scenarios for $5 \mathrm{G}$ networks.

When the cell sites in the cluster were all fully loaded; the results showed that implementing C-NOMA can decrease the cells capacity instead of increasing it. From the UEs perspective, under such circumstances, there was a $20 \%$ probability of increasing the throughput, and a $57 \%$ probability of decreasing it. Therefore, for scenarios with all equally and fully loaded cells the implementation of NC-NOMA is preferred. Future research work that proposes other methods to keep improving the system capacity gain already offered by NC-NOMA over OMA is highly anticipated.

\section{REFERENCES}

[1] L. Dai, B. Wang, Y. Yuan, S. Han, C. I, and Z. Wang, "Non-orthogonal multiple access for 5G: solutions, challenges, opportunities, and future research trends," IEEE Commun. Mag., vol. 53, no. 9, pp. $74-81,2015$.

[2] Z. Ding, Y. Liu, J. Choi, Q. Sun, M. Elkashlan, C.-L. I, and H. V. Poor, "Application of Non-orthogonal Multiple Access in LTE and 5G Networks," IEEE Commun. Mag., vol. 55, no. 2, pp. 185-191, 2017.

[3] Y. Saito, Y. Kishiyama, A. Benjebbour, T. Nakamura, A. Li, and K. Higuchi, "Non-orthogonal multiple access (NOMA) for cellular future radio access," IEEE Veh. Technol. Conf., pp. 0-4, 2013.

[4] “3GPP TR 36.859: Study on Downlink Multiuser Superposition Transmission (MUST) for LTE (Release 13)," no. V13.0.0. 2016.

[5] A. S. Marcano and H. L. Christiansen, "Performance of Non-Orthogonal Multiple Acess in mmWave wireless communications for $5 \mathrm{G}$ networks," in IEEE ICNC, 2017.

[6] A. S. Marcano and H. L. Christiansen, "A novel method for improving the capacity in 5G mobile networks combining NOMA and OMA," in IEEE 85th Vehicular Technology Conference, 2017.

[7] A. Benjebbour, Y. Saito, Y. Kishiyama, A. Li, A. Harada, and T. Nakamura, "Concept and Practical Considerations of Non-orthogonal Multiple Access (NOMA) for Future Radio Access," in International Symposium on Intelligent Signal Processing and Communications Systems, 2013.

[8] Z. Ding, Z. Yang, P. Fan, and H. V. Poor, "On the Performance of NonOrthogonal Multiple Access in 5G Systems with Randomly Deployed Users," IEEE Signal Process. Lett., vol. 21, no. 12, pp. 1501-1505, 2014.

[9] X. Xiong, W. Xiang, K. Zheng, H. Shen, and X. Wei, “An Open Source SDR-Based NOMA System for 5G Networks," IEEE Wirel. Commun., vol. 22, no. 6, pp. 24-32, 2015.

[10] "3GPP TR 36.392: Scenarios and requirements for small cell enhancements for E-UTRA and E-UTRAN (Release 13)," no. V13.0.0, 2015 .

[11] A. Benjebbour, A. Li, Y. Kishiyama, H. Jiang, and T. Nakamura, "System-Level Performance of Downlink NOMA Combined with SUMIMO for Future LTE Enhancements," in Globecom Workshops, 2014.

[12] S. Sesia, M. Baker, and I. Toufik, he UMTS Long Term Evolution: From Theory to Practic. Wiley, 2009.

[13] T. S. Rappaport, R. W. Heath Jr., C. R. Daniels, and N. J. Murdock, Millimeter Wave - Wireless Communications. Prentice Hall, 2014. 